\title{
EFEITOS DO NÚMERO DE EXEMPLARES SOBRE AQUISIÇÃO E GENERALIDADE DE DESEMPENHO EM CRIANÇAS DIAGNOSTICADAS COM TRANSTORNO DO ESPECTRO DO AUTISMO (TEA)
}

\section{EFFECTS OF THE NUMBER OF EXEMPLARS ON ACQUISITION AND GENERALITY OF PERFORMANCE CHILDREN DIAGNOSED WITH AUTISM SPECTRUM DISORDER (ASD).}

\author{
ÁlVARO JÚNIOR MELO E SILVA \\ ELIENE VAsconcelos Amaral
}

Adriano Alves BARBoza

ROMARIZ DA SILVA BARROS

UNIVERSIDADE FEDERAL DO PARÁ, BRASIL

$\mathrm{E}$

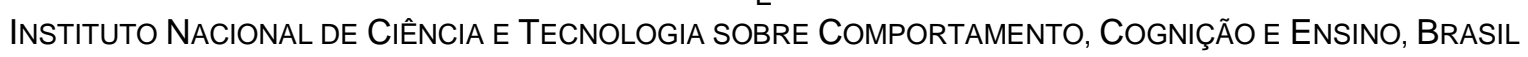

\section{RESUMO}

Considerando a importância da generalidade, uma das sete dimensões da Análise do Comportamento Aplicada, o objetivo do presente estudo foi comparar a aquisição e o grau de generalização de repertórios verbais de tacto e intraverbais quando treinados com exemplar único de estímulo antecedente e com múltiplos exemplares de antecedentes, com duas crianças diagnosticadas com Transtorno do Espectro do Autismo (TEA). Os pais das crianças foram ensinados a implementar o ensino de tacto e intraverbal via tentativas discretas (DTT). O efeito da introdução da intervenção foi avaliado por meio do delineamento de linha de base múltipla não concorrente entre participantes. O efeito de cada uma das condições experimentais foi avaliado pela comparação intrassujeito de dados entre as condições "exemplar único" versus "múltiplos exemplares" ao longo do tratamento. Assim, para uma mesma habilidade (tacto, por exemplo), algumas relações foram ensinadas com múltiplos exemplares de estímulos e outras, com exemplar único. Na fase de generalização, foi aferida a precisão de desempenho para todas as relações com novos estímulos antecedentes/exemplares. Para Guto e Manuel foram necessárias, em média, 25,5 e 15 tentativas, respectivamente, para ensinar as relações com três exemplares, e 17 e 22 tentativas para ensinar com um exemplar. Durante a fase de generalização, a precisão média nas relações com três exemplares foi 83,5\% e 100\% para Guto e Manuel, respectivamente; com um exemplar, as médias foram 74 e $61 \%$. Os dados mostram que a apresentação de múltiplos exemplares no treino de repertórios verbais proporcionou maior grau de generalização e não acarretou maior dificuldade de treino. Pelo presente estudo, encoraja-se o uso de múltiplos exemplares de estímulos em treino de repertório discriminativo em contexto aplicado.

Palavras-chave: generalização, múltiplos exemplares, repertórios verbais.

\section{ABSTRACT}

Considering the importance of generality, one of the seven dimensions of Applied Behavior Analysis, the goal of the present study was to compare the acquisition and the generalization degree of tact and intraverbal repertoires when trained with single exemplar antecedent stimulus versus multiple-exemplar antecedent stimuli, with two children diagnosed with Autism Spectrum Disorder (ASD). The children's parents were taught on how to implement these repertoires via Discrete Trial Teaching (DTT). The effect of the introduction of the intervention was evaluated by a nonconcurrent multiple baseline design between participants. The effect of each experimental condition was evaluated by comparing data from "single exemplar" versus "multiple exemplar" conditions throughout training. Then, for a given repertoire (tact, for example), some relations were taught with multiple exemplars while others, with only one exemplar. On the generalization phase, performance accuracy in all discriminations was measured with new exemplars/antecedent stimuli. For Guto and Manuel it was necessary, on average, 25.5 and 15 trials, respectively, to teach the relations with three exemplars, and 17 and 22 trials to teach with one exemplar. During the generalization phase, the average accuracy in the relations with three exemplars was $83.5 \%$ and $100 \%$ for Guto and Manuel, respectively; with one exemplar, the averages were 74 and $61 \%$. The data showed that the multiple-exemplar presentation during training of verbal repertoires resulted in higher generalization degree without increasing training difficulty. The present study encourages multipleexemplar training in applied context.

Keywords: generalization, multiple exemplars, verbal repertoires.

O trabalho é parte do programa de pesquisas do Instituto Nacional de Ciência e Tecnologia sobre Comportamento, Cognição e Ensino (INCT/ECCE), financiado pelo Conselho Nacional de Ciência de Tecnologia (CNPq - Processos \#573972/2008-7 e \#465686/2014-1) e pela FAPESP (Processos \# 08/57705-8 e \#2014/50909-8).

Endereço para correspondência: Tv. Dr. Enéas Pinheiro, 1602, apto 5. Bairro: Marco. Belém/PA. Cep: 666095-105. Email: alvarojunior.4@hotmail.com 
A Análise do Comportamento Aplicada é o ramo da Análise do Comportamento dedicado a aplicações do conhecimento e está firmemente calcada em sete dimensões, a saber: aplicada, comportamental, analítica, tecnológica, conceitualmente sistemática, efetiva e uma última dimensão, chamada generalidade (Baer, Wolf, \& Risley, 1968). Uma intervenção analítico-comportamental contempla esta última dimensão quando a mudança comportamental em questão se sustenta em uma variedade de novos ambientes, na interação com novas pessoas e em contextos diferentes daquele em que foi estritamente estabelecida (Stokes \& Baer, 1977). Adicionalmente, a mudança comportamental pode se estender a outros comportamentos. A atenção à dimensão de generalidade da intervenção também pode se relacionar ao fato de que o comportamento relevante se sustente ao longo do tempo, para além do período de intervenção (fato comumente referido como "manutenção"). Uma vez que, em função da intervenção, relações funcionais são estabelecidas também no ambiente natural - e consequências naturais facilitam sua manutenção - a intervenção geraria um ganho comportamental permanente.

O termo "generalidade" está aqui sendo empregado, portanto, em sentido diferente da generalização primária de estímulos (Keller \& Schoenfeld, 1950; Skinner, 1953). Generalização primária de estímulos refere-se ao fato de que o responder a estímulos fisicamente diferentes de um determinado estímulo discriminativo é tão mais provável quanto mais semelhante esses novos estímulos forem daquele estímulo discriminativo original, de tal forma que, ao longo de um gradiente de variações do estímulo se observa um gradiente de respostas.

Generalização, enquanto ocorrência da dimensão "generalidade", no contexto da análise comportamental aplicada, pode e deve ser programada ao longo do treino, em oposição a uma concepção do tipo "train and hope", em que se ensina um repertório e se espera que ele seja generalizável (Stokes \& Baer, 1977). A dimensão "generalidade" é, portanto, resultado da ênfase em determinadas características do procedimento de ensino (Baer et al., 1968).

Apesar de haver total concordância entre analistas do comportamento em relação à importância da dimensão "generalidade" da intervenção analítico-comportamental, nem sempre os procedimentos de intervenção incluem características que promovam generalização do desempenho em foco. Em alguns casos, a generalização do desempenho para novos ambientes, pessoas ou estímulos ocorre na ausência de programação adicional (Correa Gómez, 2015; LeBlanc et al., 2003; Wacker, Berg, Berrie, \& Swatta, 1985). Em outros casos, embora não haja uma programação formal para promover a generalização dos repertórios ensinados, os participantes são submetidos a constantes testes de generalização (Brodhead, Higbee, Gerencser, \& Akers, 2016; Dupuis, Lerman, Tsami, \& Shireman, 2015; Farber, Dube, \& Dickson, 2016; Gruber \& Poulson, 2016; Jeffries, Crosland, \& Miltenberger, 2016). Adicionalmente, há relatos de estudos em que não houve programação formal para generalização e, quando testada, os resultados foram negativos (Austin \& Tiger, 2015; Coelho, 1990; Hine, Ardoin, \& Foster, 2015; Neef, Walters, \& Egel, 1984; Shillingsburg, Kelley, Roane, Kisamore, \& Brown, 2009). Os estudos não esclarecem as variáveis responsáveis por tal variabilidade de resultados.

LeBlanc et al. (2003), por exemplo, utilizaram um procedimento de videomodelação e reforçamento para ensinar habilidades de tomada de perspectiva a três crianças com autismo. Ao final do treino, foram realizados testes de generalização, porém os autores relatam que o nível de generalização foi limitado. Uma das possíveis limitações do estudo foi a ausência de treino em situações sociais naturais. Isso pode ter sido um fator determinante para a limitação do desempenho dos participantes nos testes de generalização.

Generalização não é, portanto, um processo passivo. É possível, e desejável, criar condições de forma a aumentar sua probabilidade e não apenas esperar pela ocorrência deste fenômeno comportamental (Silva, 2015; Stokes \& Baer, 1977). Nesse sentido, a introdução de certo nível de variação (de pessoas aplicando os procedimentos, de contextos e de estímulos) ao longo do treino do repertório-alvo é uma estratégia central para a promoção de generalização. A utilização de múltiplos exemplares de estímulos, por exemplo, tem sido de fundamental importância no planejamento da generalização (Baer 1981; Stokes \& Baer, 1977).

Na literatura, há exemplos de comparação de métodos de ensino, que são discutidos em termos de estabelecimento de repertórios, bem como de sua generalização (e.g., Cuvo et al., 1980). No estudo de Cuvo et al., um delineamento fatorial foi utilizado para comparar a eficácia de métodos de apresentação de estímulos (sucessivo, simultâneo e combinado) no ensino de nomeação. Neste caso, os métodos Simultâneo e Combinado foram considerados mais eficazes. Entretanto, os autores discutem que o modelo simultâneo seria mais eficaz para a produção de generalização.

Há alguns estudos na literatura que obtiveram resultados positivos de generalização do desempenho utilizando múltiplos exemplares de estímulos durante o ensino (Albright, Reeve, Reeve, \& Kisamore, 2015; Dixon, Peach, \& Daar, 2017; Fiorile \& Greer, 2007; Frampton, Wymer, \& Hansen, 2016; MacDonald \& Ahearn, 2015; Marzullo-Kerth, Reeve, Reeve, \& Townsend, 2011; Reeve, Reeve, Townsend, \& Poulson, 2007; Silber \& Martens, 2010). Devido às suas características, o ensino com múltiplos exemplares já prevê tais resultados devido à programação para a generalização desde suas fases iniciais.

O potencial aumento da dificuldade de treino com a introdução de múltiplos exemplares de estímulos pode se constituir como uma barreira para a adoção desse e de outros tipos de estratégias para promover generalização. Essa questão não foi diretamente enfocada pelos estudos anteriores, mas é particularmente importante quando a implementação de procedimentos de ensino é feita por cuidadores. Assim, é possível levantar a questão sobre o 
quão mais difícil se torna o estabelecimento de um determinado repertório quando ele se dá em condições de variações de exemplares (e, portanto, em condições favoráveis à generalização). Embora esse tipo de estratégia possa favorecer a generalização, é importante que ela não torne a implementação da intervenção, e os efeitos de aprendizagem, mais difíceis de se alcançar. Isso é particularmente verdadeiro na intervenção implementada por cuidadores, onde a implementação é, em grande medida, feita por leigos (para pesquisas no Brasil sobre a intervenção implementada por cuidadores, ver Barboza, Silva, Barros, \& Higbee, 2015; Borba, Monteiro, Barboza, Trindade, \& Barros, 2015; Ferreira, Silva, \& Barros, 2016; Silva, 2015). Este tipo de intervenção favorece a generalização dos repertórios estabelecidos para outros ambientes, diante de diferentes indivíduos e seria ainda mais fortalecido com a inserção de procedimentos que promovam ainda mais a generalização dos repertórios aprendidos (e.g., variações de exemplares).

Considere, por exemplo, que uma determinada criança com atraso severo no desenvolvimento esteja sendo submetida ao ensino de repertório intraverbal com foco em informações pessoais. Um dos repertórios-alvo seria o de dizer seu próprio nome quando perguntado. Assim, seria apresentado como estímulo antecedente a pergunta “Qual é o seu nome?". A resposta-alvo seria dizer o próprio nome (por exemplo, dizer “José"). A resposta então seria reforçada. $\mathrm{O}$ uso de múltiplos exemplares de estímulos determinaria que, já desde o início do treino, além do antecedente "Qual é o seu nome?", outros exemplares de estímulos antecedentes deveriam ser apresentados, como "Como você se chama?" e "Como é o seu nome?". Assim, em um teste subsequente de generalização, seria mais provável que a resposta-alvo (dizer "José") ocorra quando um novo estímulo antecedente for apresentado ("Seu nome é...").

De maneira geral, os estudos têm como objetivo estabelecer, em cuidadores, repertórios que possibilitem adotar diferentes procedimentos de ensino junto a seus filhos com TEA. São adotados pacotes de ensino com diferentes procedimentos (p. ex., videomodelação, múltiplos exemplares, legendas, role-play). Esses estudos relatam sucesso na instalação dos repertórios nos cuidadores, na aprendizagem pelas crianças por eles treinadas e em generalização. Barboza et al. (2015) ensinaram três cuidadores por vídeo-modelação a aplicar um programa de ensino de habilidades comportamentais e verificaram, por um delineamento de linha de base múltipla entre participantes, a efetividade do ensino. Ferreira et al. (2016) ensinaram cinco pais a implementarem ensino por tentativas discretas (DTT) para uma série de habilidades de suas crianças com TEA. Um delineamento quase experimental verificou, nos pós-testes, que os participantes alcançaram $100 \%$ de precisão. Esses estudos, embora relatem o estabelecimento do repertório desejado no cuidador, não exploraram diretamente o potencial de procedimentos para promover a generalidade dos comportamentos nem explicitaram o quão mais demorado foi o ensino (com procedimentos de variações de exemplares), nem o quanto mais de generalização se obteve, com e sem procedimentos de promoção de generalização.

Sendo assim, o objetivo do presente estudo foi comparar a aquisição, e o grau de generalização, de repertórios verbais quando ensinados com exemplar único de estímulo antecedente e com múltiplos exemplares de antecedentes, com crianças diagnosticadas com TEA, em que o ensino foi conduzido por cuidadores. O arranjo de contingências adotado foi o ensino por tentativas discretas (do inglês, Discrete Trial Teaching - DTT), conduzido por cuidadores de crianças com diagnóstico de Transtorno do Espectro do Autismo (TEA). O DTT é uma forma de estruturar o ensino de modo sistemático, planejado e controlado (Wong et al., 2014). O instrutor controla a apresentação dos estímulos antecedentes, aguarda a resposta e apresenta consequências de acordo com o planejado. Foram selecionados repertórios verbais a serem ensinados às crianças (conforme uma avaliação estruturada prévia). Então, os pais das crianças foram treinados a implementar ensino desses repertórios via DTT, com múltiplos exemplares de estímulos para alguns repertórios e com exemplar único para outros.

\section{MÉTODO}

\section{Participantes}

Dois pais e suas respectivas crianças participaram do estudo: Flávia (36 anos de idade) e seu filho Guto (5); e Mário (32) e seu filho Manuel (7). Os nomes utilizados para os participantes são fictícios com o objetivo de preservar a identidade dos mesmos. As crianças faziam parte de um programa de ensino de cuidadores no Projeto APRENDE (Atendimento e Pesquisa sobre Aprendizagem e Desenvolvimento, Universidade Federal do Pará). Foram realizadas avaliações comportamentais das duas crianças, com base no Verbal Behavior Milestones Assessment (VBMAPP) (Sundberg, 2014). O VBMAPP é um instrumento de avaliação principalmente de habilidades verbais e guia curricular. O instrumento é dividido em 5 partes, a saber: marcos, barreiras, transição, análise de tarefas e plano de ensino individualizado. No presente estudo, apenas algumas habilidade dos marcos foram avaliadas. Os marcos têm as habilidades divididas ao longo de três níveis (Nível 1, Nível 2 e Nível 3). O Nível 3 é composto pelas habilidades mais complexas. Guto teve a maioria das suas habilidades situadas no Nível 1 (equivalente a 0-18 meses) e Manuel no Nível 2 (equivalente a 18-30 meses) do instrumento. Guto tacteava dez itens quaisquer (objetos comuns, pessoas, partes do corpo etc), mas não tacteava ações. Guto emitia mandos sem o auxílio de dicas e na presença do item demandado; a resposta de ouvinte mais complexa era selecionar um item correto em um conjunto de 4 itens; além disso, imitava movimentos motores de vários tipos. Manuel, com relação ao operante verbal intraverbal, completava dez diferentes frases preenchendo as lacunas de qualquer tipo (ex.: completar música, brincadeiras sociais, preenchimento de lacunas divertidas, sons de animal e de objetos); mas não respondia a questões pessoais (seu nome, por exemplo); quanto a tacto, o repertório mais complexo era nomear itens ou ações; emitia mandos com duas ou mais palavras; 
seguia instruções de dois componentes substantivo-verbo e/ou verbo substantivo e, quanto à imitação, imitava movimentos de qualquer tipo. Como resultado da avaliação, foi determinado que, na presente pesquisa, Manuel seria submetido a ensino de repertório intraverbal (informações pessoais) e Guto a treino de tacto (ações). Outros alvos de intervenção ficaram sob enfoque de outras iniciativas de pesquisa e intervenção dentro do projeto APRENDE e ocorriam antes ou após a condução do presente experimento. Os pais das crianças participaram do estudo de Barboza et al. (2015), no qual aprenderam a aplicar o ensino por tentativas discretas (DTT), a implementar procedimentos de ajuda e de correção. Dois dias antes do começo do presente estudo, ambos os cuidadores tiveram seus desempenhos avaliados e apresentaram precisão de desempenho acima de $85 \%$ na implementação de DTT. No decorrer do estudo, o desempenho de aplicação do cuidador foi acompanhado por meio da avaliação da integridade do procedimento (era avaliada uma sessão a cada três). Quando se detectava precisão inferior a $85 \%$, os procedimentos de feedback do estudo de Barboza et al. (2015) eram fornecidos, com o intuito de aumentar a precisão de desempenho do cuidador. Os participantes relataram pertencer às classes sócio-econômicas média-baixa (Flávia) e baixa (Mário). As sessões experimentais duravam entre 10 e 15 minutos e começaram a ser implementadas concomitantemente na universidade e em casa, sendo a primeira sessão realizada na universidade. Para Guto, a média de ocorrência de sessões, por semana, foi 6,6 em casa e 1,6 na universidade. Para Manuel, a média foi de 3,6 em casa e 2,6 na universidade.

\section{Ambiente e materiais}

$\mathrm{Na}$ universidade, as sessões experimentais eram conduzidas em uma sala de pesquisa $(2,5 \times$ X 2,5 m²), com iluminação artificial e ambiente climatizado. Neste espaço, havia uma mesa com duas cadeiras (uma para a criança e outra para o cuidador), uma terceira cadeira para o experimentador, que acompanhava as sessões, uma caixa com brinquedos e alimentos, ao alcance do cuidador. Estes brinquedos e alimentos, que foram utilizados como consequência para acertos da criança, eram selecionados no início de cada sessão, quando o experimentador disponibilizava para a criança diversos itens e separava, para usar na sessão experimental, aqueles que ela selecionava com maior frequência.

Foram utilizadas folhas de registro e lápis pelo cuidador para registrar as respostas-alvo da criança. As sessões experimentais também foram conduzidas pelos cuidadores em suas residências, sem a presença do experimentador. Apenas com Guto foi utilizado um tablet para apresentar os estímulos do seu programa de ensino. Estes estímulos consistiam de imagens em movimento $(G I F)$, de alguém realizando alguma ação (na Figura 1, pode ser visto um print dos referidos GIFS). Uma câmera filmadora foi utilizada para gravar, com ambos os participantes, as sessões realizadas na universidade. As filmagens foram utilizadas para a avaliação do acordo entre observadores com relação à fidedignidade do registro dos dados e avaliação da integridade da implementação do procedimento.

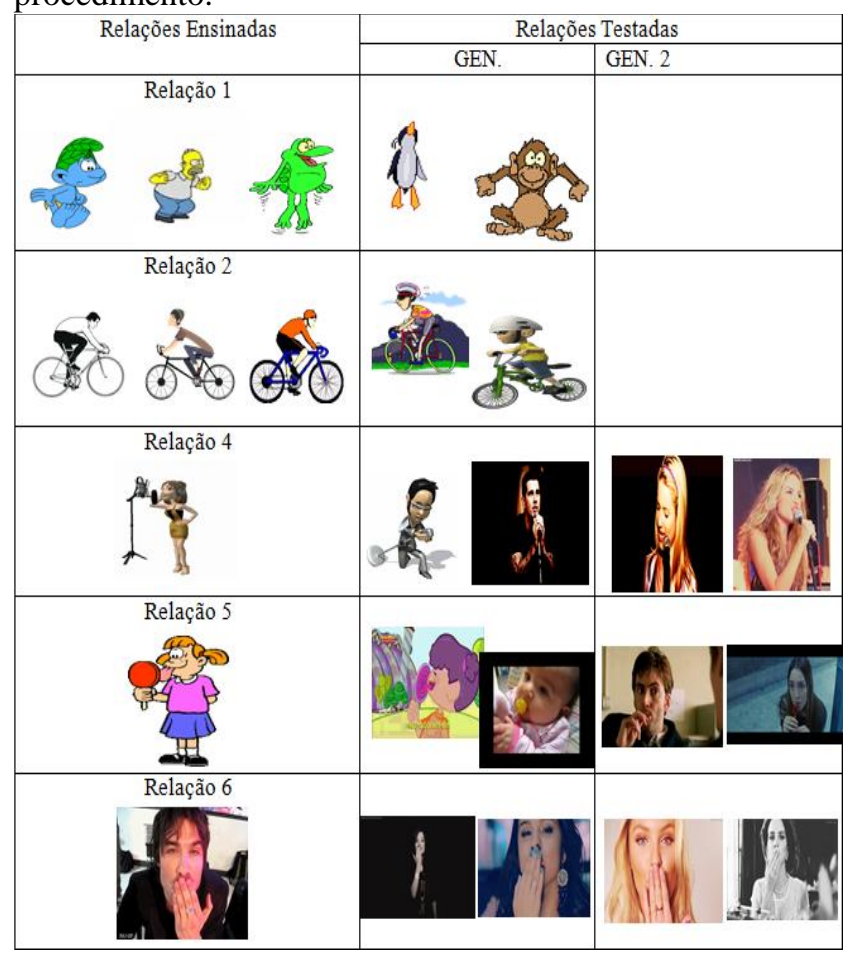

Figura 1. Relações de tacto ensinadas e testadas para o participante Guto.

\section{Variável dependente}

A variável dependente foi a precisão do desempenho nas tentativas discretas de repertório verbal aferida pela percentagem de respostas vocais corretas, sem prompt. Por exemplo, em uma tentativa de ensino de intraverbal (com Manuel), na presença do antecedente verbal "Quantos anos você tem?", o participante deveria dizer "7 anos". Em uma tentativa de tacto (com Guto), quando apresentada a imagem de uma pessoa andando de bicicleta, o participante deveria dizer "andando de bicicleta".

\section{Acordo entre observadores e integridade de implementação do procedimento}

Foram aferidos o acordo entre observadores e a integridade de implementação do procedimento para $30 \%$ do total de sessões (uma sessão a cada três, selecionada de forma aleatória), incluindo todas as etapas do experimento (linha de base, intervenção, generalização e follow-up). A única exceção foi o follow-up para Guto, cuja sessão, por problemas técnicos, não foi gravada. O acordo entre observadores foi realizado com base no registro do cuidador e de um observador treinado (experimentador). A avaliação da integridade do procedimento foi conduzida pelo experimentador. Com relação ao registro dos dados, foi considerado um acordo quando tanto o cuidador quanto o experimentador registraram "resposta correta sem prompt", "resposta correta com prompt" ou "resposta errada" para uma mesma resposta do participante. O acordo entre observadores foi obtido dividindo-se o número de acordos pelo somatório de acordos e desacordos e multiplicando esse total por 100. A média de 
acordo entre observadores para Guto foi $96,6 \%$ (98\% na linha de base, $92 \%$ no treino com um ou três exemplares e $100 \%$ na generalização) e para Manuel 98,5\% (100\% na linha de base, $100 \%$ no treino com um ou três exemplares, $100 \%$ na generalização e $94 \%$ no follow-up).

A avaliação da integridade da aplicação do procedimento foi realizada levando-se em consideração a implementação de cada tentativa de ensino, exclusivamente nas sessões conduzidas no ambiente do laboratório. Foi avaliado, de acordo com um protocolo previamente estabelecido, se o experimentador: 1) obteve a atenção da criança; 2) apresentou o estímulo antecedente corretamente; 3) forneceu prompt/reforçou/corrigiu corretamente a resposta da criança e a registrou. A integridade do procedimento foi obtida dividindo-se o número de itens implementados corretamente em cada tentativa pelo total de itens das tentativas e multiplicando por 100. A média de integridade do procedimento para Flávia foi $89,3 \%$ (100\% na linha de base, $84 \%$ no treino com um ou três exemplares e $84 \%$ na generalização) e $100 \%$ para Mário.

\section{Delineamento Experimental}

Foi utilizado um delineamento de linha de base múltipla não concorrente entre participantes. A variável independente (VI) deste estudo foi a implementação do procedimento de treino, que apresentava duas variações quanto à quantidade de estímulos antecedentes para cada resposta-alvo: um ou três. Após a condução de cinco sessões de linha de base para o participante Guto, a VI foi implementada. Dez sessões de linha de base foram conduzidas para o participante Manuel para, então, a VI ser implementada. As coletas foram conduzidas em momentos diferentes para os dois participantes. O efeito da intervenção foi avaliado mediante o delineamento de linha de base múltipla. $\mathrm{O}$ efeito diferencial das duas variações da VI foi aferido por alternâncias de tratamento ao longo da introdução da VI: para um mesmo participante, alguns alvos eram ensinados com três exemplares de antecedentes e outros com um exemplar em etapas subsequentes do treino.

\section{Procedimento} entrada.

Linha de base: avaliação do repertório de

O objetivo desta fase foi aferir a precisão de desempenho inicial da criança em cada relação do programa de ensino selecionado. Assim como na fase de ensino, as sessões de linha de base foram conduzidas no formato de ensino por tentativas discretas, no entanto, não houve consequência diferencial para as respostas-alvo. A cada duas tentativas, em média, uma demanda alternativa era apresentada (toque aqui, por exemplo) e um estímulo de preferência da criança (brinquedo ou comestível) era entregue à mesma, com o objetivo de mantê-la engajada na tarefa. Estas sessões foram realizadas em diferentes dias da semana e tanto pelo cuidador, quanto pelo experimentador. Todos os estímulos antecedentes das relações do programa de ensino foram apresentados nesta fase.

\section{Treino com um e com três exemplares.}

Um programa de ensino para cada criança foi selecionado: "Informações Pessoais" para Manuel e "Tacto de Ações" para Guto. Para o programa "Informações Pessoais" foram selecionadas quatro relações intraverbais e para o programa "Tacto de Ações" foram selecionadas seis relações de tacto, no entanto, uma relação foi removida no decorrer do treino (ver Figura 1). Em cada programa de ensino, para metade das relações, foram selecionados três diferentes exemplares (por exemplo, "Como sua mãe se chama?", "O nome da sua mãe é?" e "Sua mãe se chama...". Para outra metade, apenas um exemplar foi definido (por exemplo, "Onde você estuda?") (Ver Tabela 1). Para Manuel, as sessões foram compostas de oito tentativas (três tentativas para cada uma das duas relações intraverbais com três exemplares, uma tentativa para cada exemplar; e uma tentativa para cada relação intraverbal com um exemplar). Para Guto, as sessões eram compostas por doze tentativas e, também, $3 / 4$ das tentativas eram para as relações com três exemplares. Tanto a quantidade de relações por programa de ensino, quanto a quantidade de exemplares por tipo de relação (um e três) foram definidas de forma arbitrária. As relações para as quais foram empregados um ou três exemplares na fase de ensino foram definidas por sorteio.

\section{Sessões de Generalização (com novos exemplares) e follow-up.}

Quando o desempenho dos participantes atingiu critério de precisão (100\% em duas sessões consecutivas) em todas as relações na fase de treino, foi conduzida a fase de generalização. O objetivo desta fase consistiu em aferir a precisão de desempenho nas relações (com um e com três antecedentes/exemplares na fase de ensino), com novos estímulos antecedentes/exemplares. Assim, foram adicionados estímulos que não fizeram parte do treino, mas que possuíam semelhanças físicas com os mesmos. No programa de "Informações pessoais", por exemplo, foram inseridos dois exemplares ("Quantos anos você tem?" e "Sua idade é...") para a classe de estímulos que já continha na fase de treino ("Qual a sua idade?", "Que idade você tem?" e "Você tem quantos anos?"). Para as relações que continham um exemplar na fase de treino (por exemplo, "Qual o nome da sua mãe?"), quatro novos exemplares foram adicionados ("Como sua mãe se chama?", "O nome da sua mãe é...", "Sua mãe se chama...", “Que nome tem sua mãe?") (ver Tabela 1). Quando erros ocorreram, foram seguidos pelo procedimento de correção. Este procedimento consistiu em: retirar a atenção por 3 segundos; reapresentar o estímulo antecedente e fornecer prompt (experimentador apresentou a resposta vocal alvo para que a criança repetisse); apresentar uma demanda de baixo custo e de domínio da criança (bate palmas, por exemplo); reapresentar tentativa corrente dando a oportunidade de ocorrer resposta sem prompt e fornecer elogio contingente à resposta. 
Tabela 1.

Relações Intraverbais Ensinadas e Testadas para o Participante Manuel.

\begin{tabular}{|c|c|c|c|c|}
\hline \multirow[t]{2}{*}{ Relações Ensinadas } & \multicolumn{4}{|c|}{ Relações Testadas } \\
\hline & GEN 1 & GEN 2 & GEN 3 & GEN 4 \\
\hline $\begin{array}{l}\quad \text { Relação } 1 \\
\text { 1. Qual a sua idade? } \\
\text { 2. Que idade você tem? } \\
\text { 3. Você tem quantos anos? }\end{array}$ & 4. Quantos anos você tem? & 5. Sua idade é? & & \\
\hline $\begin{array}{l}\text { Relação } 2 \\
\text { 1. Onde você estuda? } \\
\text { 2. Você estuda na escola? } \\
\text { 3. Qual o nome da sua escola }\end{array}$ & $\begin{array}{l}\text { 4. Você estuda em qual } \\
\text { escola? }\end{array}$ & 5. O nome da sua escola é? & & \\
\hline $\begin{array}{l}\text { Relação } 3 \\
\text { 1. Seu nome é? }\end{array}$ & 2. Qual o seu nome? & 3. Como você se chama? & 4. Você se chama? & 5. Que nome você tem? \\
\hline $\begin{array}{c}\text { Relação } 4 \\
\text { 1. Qual o nome da sua mãe? }\end{array}$ & 2. Como sua mãe se chama? & 3. 0 nome da sua mãe é? & 4. Sua mãe se chama? & 5. Que nome tem a sua mãe? \\
\hline
\end{tabular}

Os estímulos foram selecionados aleatoriamente para compor a fase de ensino ou de generalização. Para as relações intraverbais, os novos exemplares foram adicionados um de cada vez e a inserção de um próximo exemplar nem sempre foi condicionada à precisão de $100 \%$ na relação com o exemplar corrente (por falha na programação experimental). Para as relações de tacto, os novos exemplares foram adicionados dois de cada vez e a inserção de novos exemplares dependeu de $100 \%$ de precisão nas relações com os exemplares correntes. A sessão de follow-up foi programada para ocorrer um mês após a última sessão de generalização e incluiu todos os exemplares dos programas. Tanto as sessões de generalização, quanto as de follow-up, foram planejadas para ocorrer apenas na universidade e conduzidas pelos cuidadores. Este planejamento foi possível apenas com o participante Guto. O participante Manuel não pôde ir algumas vezes à universidade, então, orientações foram passadas e algumas sessões de generalização foram conduzidas em casa. Pelo menos duas sessões de generalização das conduzidas em casa foram acompanhadas e gravadas pelo experimentador e compuseram a amostra para a avaliação do acordo entre observadores.

\section{RESULTADOS E DISCUSSÃO}

Os dados do presente estudo mostraram que ambos os participantes aprenderam todas as relações que haviam sido programadas em todas as fases de treino, com exceção da Relação 3 para Guto. A Figura 2 apresenta a precisão de desempenho (percentagem de respostas corretas sem prompt) ao longo da Linha de Base (LB), Treino Com Um e Com Três Exemplares, Generalização (GEN.) e Follow-up (F-U) para os participantes Guto e Manuel. Nas fases de LB, Treino e Generalização, quadrados vazios pretos apresentam o desempenho em relações com três exemplares e triângulos pretos preenchidos apresentam o desempenho em relações com um exemplar. Nas fases de Treino e Generalização, esses marcadores representam sessões realizadas na casa do cuidador. As sessões de LB foram todas realizadas na universidade. Nas fases de Treino, Generalização e Follow-up, círculos cinza vazios apresentam o desempenho em relações com três exemplares e círculos cinza preenchidos apresentam o desempenho em relações com um exemplar em sessões realizadas na universidade.

A partir da Figura 2, comparando o desempenho nas relações alvo na linha de base (precisão de desempenho igual a zero) com o desempenho na fase de Treino, é possível verificar que houve aumento na precisão de desempenho imediatamente após a introdução da intervenção, com a precisão de desempenho ascendente (embora variável) até o estabelecimento das relações, tanto com um quanto com três exemplares de estímulos. Os dados de generalização e follow-up também permitem constatar efeito da intervenção.

Para além de avaliar, por meio do delineamento de linha de base múltipla, se a intervenção implementada produziu efeitos sobre a variável dependente, o presente estudo enfoca, pela alternância de tratamentos durante a intervenção, se houve ou não diferenças na aquisição e generalização de repertórios entre as modalidades de ensino com um e três estímulos antecedentes. Assim, a Figura 3 apresenta os dados de forma mais detalhada, o que permite abordar mais precisamente essa pergunta. Pode-se observar, na Figura 3, a precisão de desempenho (percentagem de respostas corretas sem prompt) ao longo da Linha de Base (LB), Treino Com Um e Três Exemplares, Generalização (GEN, GEN 1 e GEN 2 para Guto e GEN 1, GEN 2, GEN 3 e GEN 4 para Manuel) e Follow-up (F-U) para Guto (Relações 1, 2, 4, 5 e 6) e para Manuel (Relações 1, 2, 3 e 4). Círculos pretos representam os dados da intervenção conduzida na universidade e triângulos cinza representam os da intervenção em casa. 


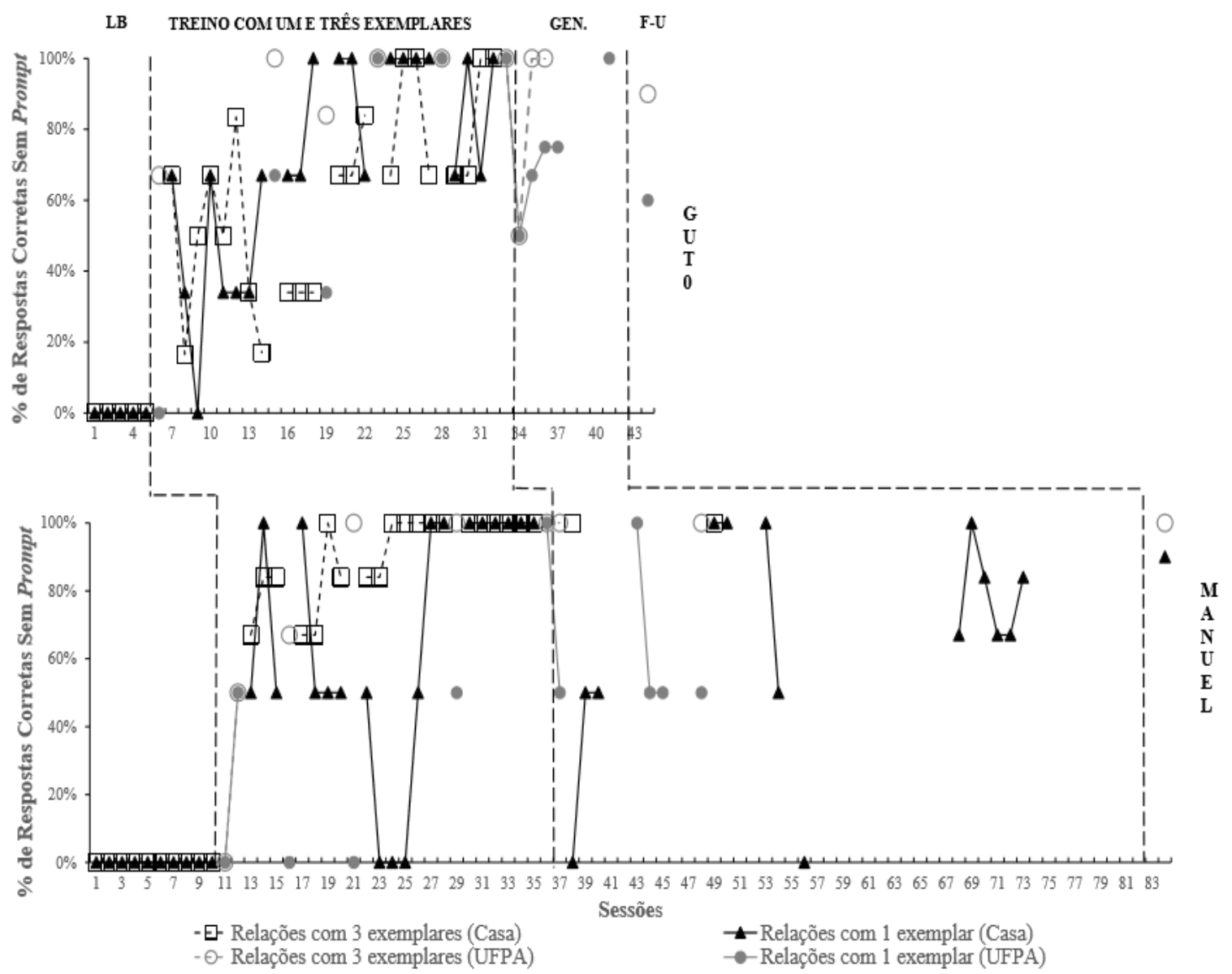

Figura 2. Percentagem de respostas corretas sem prompt, para Guto e Manuel, ao longo das fases de Linha de Base (LB), Treino Com Um e Com Três Exemplares, Generalização e Follow-up (F-U). Nas fases de LB, Treino e Generalização, quadrados vazios pretos apresentam o desempenho em relações com três exemplares e triângulos pretos preenchidos apresentam o desempenho em relações com um exemplar. Nas fases de Treino, Generalização e Follow-up, círculos cinza vazios apresentam o desempenho em relações com três exemplares e círculos cinza preenchidos apresentam o desempenho em relações com um exemplar em sessões realizadas na universidade.

A Relação 3 foi retirada do treino do participante Guto, pois este não tacteava de forma inteligível a ação, além de que, já na fase de linha de base, foram verificadas algumas topografias de repostas semelhantes às que o participante passou a emitir diante da ação durante a fase de treino.

A precisão média durante as sessões de linha de base foi zero em todas as relações, para os dois participantes. Guto foi submetido a cinco sessões de linha de base para todos os repertórios, enquanto Manuel foi submetido a 10 sessões.

A precisão média durante o "Treino com Três Exemplares" foi 68,5\% para Guto (70\% na Relação 1 e $67 \%$ na Relação 2) e $86 \%$ para Manuel (90\% na Relação 1 e $82 \%$ na Relação 2). Durante o "Treino com Um Exemplar", a precisão média foi $70,3 \%$ para Guto $(54 \%$ na Relação 4, 82\% na Relação 5 e $75 \%$ na Relação 6) e 60, 5\% para Manuel (63\% na Relação 3 e 58\% na Relação 4).

Uma avaliação sobre se o treino com mais exemplares pode acrescentar significativamente dificuldades de treino pode ser feita com base nos dados da Figura 4. Tomando-se conjuntamente todos os dados das relações ensinadas, para que se atingisse o critério de aprendizagem, foram necessárias, em média, 25,5 tentativas para as relações ensinadas com três exemplares e 17 tentativas para as relações com 1 exemplar para Guto. Com relação a Manuel, para que se atingisse o critério de aprendizagem, foram necessárias, em média, 15 tentativas para as relações ensinadas com três exemplares e 22 tentativas para as relações com 1 exemplar. Esses dados mostram que a introdução de múltiplos exemplares não acarreta necessariamente em mais demanda de treino. Com Guto, o número de tentativas processadas foi $50 \%$ maior com 3 exemplares, comparado com 1 exemplar. Com Manuel, contudo, o número de tentativas processadas foi 31,8\% menor com 3 exemplares, comparado com 1 exemplar. Os dados obtidos com Manuel são particularmente intrigantes porque mostram que é possível que o treino com múltiplos exemplares de estímulos não acrescente nenhuma dificuldade para aquisição das discriminações, ou até funcione de alguma forma como uma vantagem. 

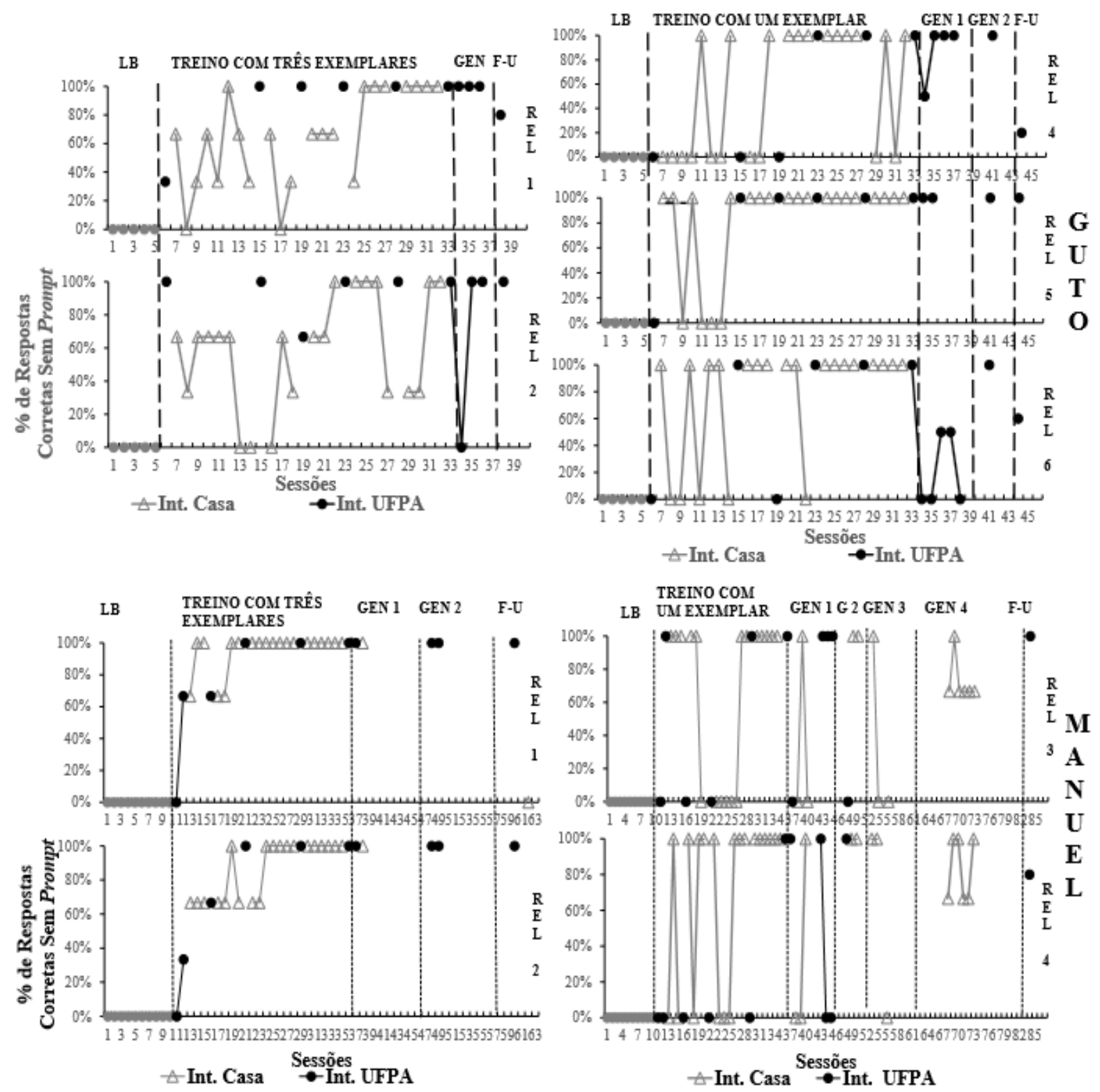

Figura 3. Percentagem de respostas corretas sem prompt, para Guto e Manuel, ao longo das fases de Linha de Base (LB), Treino Com Um e Três Exemplares, Generalização (GEN 1 e GEN 2 para Guto e GEN 1, GEN 2, GEN 3 e GEN 4 para Manuel) e Follow-up (F-U) para Guto (Relações 1, 2, 3, 4 e 5) e para Manuel (Relações 1, 2, 3 e 4) nos diferentes ambientes. Círculos pretos apresentam os dados da intervenção conduzida na universidade e triângulos cinza apresentam os da intervenção em casa.

Com relação ao grau de generalidade do desempenho ensinado com 1 ou com 3 exemplares, os dados são apresentados na Figura 5.

Durante a fase de generalização, a precisão média nas relações com três exemplares foi $83,5 \%$ (100\% na Relação 1 e $67 \%$ na Relação 2) e com um exemplar foi de $74 \%$ (90\% na Relação 4, $100 \%$ na Relação 5 e 33\% na Relação 6) para Guto. Esse dado mostra 9,5\% a mais de generalização nos desempenhos ensinados com três exemplares. Para Manuel, a precisão média com três exemplares foi de $100 \%$ e com um exemplar foi $61 \%$
(60\% na Relação 3 e 62 na Relação 4), o que mostra 39\% a mais de generalização para os repertórios ensinados com três exemplares. Durante o follow-up, a precisão média nas relações com três exemplares foi $90 \%$ (80\% na Relação 1 e $100 \%$ na Relação 2) e 60\% (20\% na Relação 4, 100\% na Relação 5 e 60\% na Relação 6) com um exemplar para Guto. Para Manuel, a precisão foi de $100 \%$ com três exemplares e $90 \%$ (100\% na Relação 1 e $80 \%$ na Relação 2) com um exemplar. Esses dados mostram $30 \%$ a mais de manutenção do desempenho com três exemplares para Guto e $10 \%$ a mais para Manuel. 


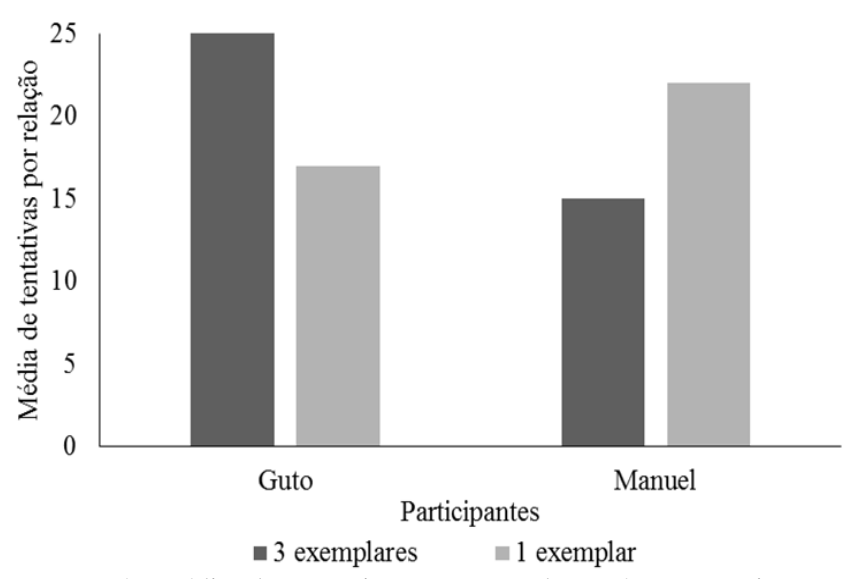

Figura 4. Média de tentativas processadas até que se tivesse obtido o critério de precisão de desempenho nas relações treinadas com 1 e com 3 exemplares, para os participantes Guto e Manuel.

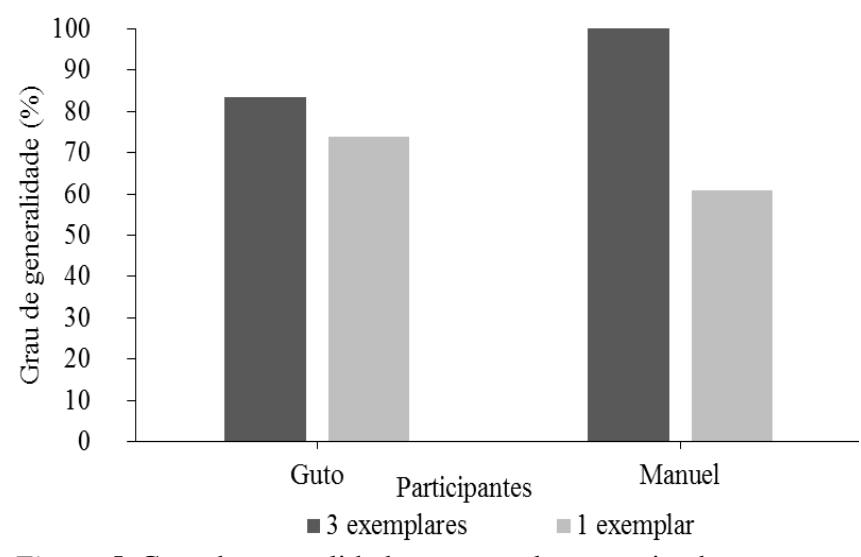

Figura 5. Grau de generalidade para as relações treinadas com um e três exemplares.

Considerando que o principal objetivo do presente estudo foi verificar o efeito da utilização de múltiplos exemplares sobre a aquisição e generalização de repertórios verbais em crianças diagnosticadas com TEA, é possível afirmar que a apresentação de múltiplos exemplares no treino de repertórios verbais pode não acarretar em maior dificuldade de treino (ver dados de Manuel). Além disso, quanto a inserção de múltiplos exemplares acarretou mais treino, a quantidade adicional de treino ficou abaixo da proporção de aumento de número de exemplares (ver dados de Guto). Adicionalmente, ganhos de generalização e manutenção foram observados com a inserção de múltiplos exemplares no treino). É possível notar, quando da inserção de novos exemplares, maior precisão de desempenho (média de $83,5 \%$ para Guto e $100 \%$ para Manuel) nas relações que possuíam três exemplares durante o treino (ver Figura 2). Ao considerarmos a primeira sessão de generalização (Sessão 34 para Guto e 37 para Manuel), quando é possível ver mais claramente o efeito dos dois tipos de treino (com um e três exemplares), a precisão média nas relações com três exemplares foi $50 \%$ (100\% na Relação 1 e $0 \%$ na Relação 2) para Guto e $100 \%$ para Manuel. Nas relações com um exemplar, a precisão média foi 50\% (50\% na Relação 4,
$100 \%$ na Relação 5 e $0 \%$ na Relação 6) para Guto e $50 \%$ (0\% na Relação 3 e 100\% na Relação 4) para Manuel. Considerando as mesmas sessões (34 para Guto e 37 para Manuel), a probabilidade de precisão máxima (100\% de acertos) nos novos exemplares foi de $75 \%$ (3/4) nas relações com três exemplares no treino e $40 \%(2 / 5)$ para as relações com um exemplar.

Os dados supracitados corroboram o argumento de Stokes e Baer (1977) e Silva (2015) de que podemos planejar condições de forma a aumentar a probabilidade de generalização de estímulos. Na ausência de programação adicional, generalização até pode ocorrer, como é visto em alguns estudos na literatura (Wacker, Berg, Berrie, \& Swatta, 1985; LeBlanc et al., 2003; Correa Gómez, 2015), mas com menor probabilidade, como visto no presente estudo. Os dados também encorajam o uso de múltiplos exemplares no ensino na medida em que favoreceram a aquisição (no caso de Manuel) ou acrescentaram uma dificuldade proporcionalmente menor que os ganhos (no caso de Guto).

Uma limitação deste estudo foi quanto ao não equilíbrio na distribuição das tentativas para os dois tipos de relação (com um e com três exemplares). Em um próximo estudo, caso sejam selecionadas seis relaçõesalvo, a sessão poderá ser composta por 18 tentativas, de forma que 9 tentativas serão para as relações com três exemplares (uma tentativa para cada um dos exemplares) e 9 tentativas para as relações com um exemplar (três tentativas para cada um dos exemplares).

Outra limitação do estudo, foi quanto à inserção de novos exemplares, para Manuel, sem o participante ter atingido critério de precisão na relação com o exemplar anteriormente adicionado. Em estudo futuro, replicações internas deverão ser conduzidas. Nesse sentido, após a inserção de novos exemplares, caso o desempenho do participante não atinja critério de precisão na primeira sessão, poderá ser conduzido um treino com os novos exemplares, até se atingir critério de precisão e, quando da inserção de novos exemplares, será observado o efeito do treino com múltiplos exemplares. Também poderá investigado se quanto maior o número de exemplares, maior é a probabilidade de generalização (por exemplo, com um, três e cinco exemplares). Um cuidado adicional deve ser tomado, quanto à seleção da habilidade. É importante que seja selecionada uma mesma habilidade para todos os participantes, pois no presente estudo a diferença entre os desempenhos dos participantes pode ter sido em função das habilidades diferentes (tacto e intraverbal).

Por último, um ponto que merece ser discutido, por talvez tratar-se de uma limitação deste estudo, é a condução do treino em diferentes ambientes sem as mesmas condições de controle. Para as relações-alvo do participante Guto, principalmente as com três exemplares de estímulo, foi detectada alta precisão de desempenho nas sessões realizadas na universidade e baixa (por algumas sessões), para as sessões realizadas em casa. Em estudos subsequentes nesta linha de pesquisas, o treino será conduzido apenas na universidade para que seja possível obter um maior controle das variáveis. 


\section{REFERÊNCIAS}

Albright, L., Reeve, K. F., Reeve, S. A., \& Kisamore, A. N. (2015). Teaching statistical variability with equivalence-based instruction. Journal of Applied Behavior Analysis, 48, 883-894. doi:10.1002/jaba.249

Austin, J. E., \& Tiger, J. H. (2015). Providing alternative reinforcers to facilitate tolerance to delayed reinforcement following Functional Communication Training. Journal of Applied Behavior Analysis, 48, 663-668. doi: 10.1002/jaba.215

Baer, D. M., Wolf, M. M., \& Risley, T. R. (1968). Some current dimensions of Applied Behavior Analysis. Journal of Applied Behavior Analysis, 1(1), 91-97. doi: 10.1901/jaba.1968.1-91

Baer, D. M. (1981). How to plan for generalization. Lawrence: H \& H Enterprises, Inc.

Barboza, A. A., Silva, A. J. M., Barros, R. S., \& Higbee, T. S. (2015). Efeitos de videomodelação instrucional sobre o desempenho de cuidadores na aplicação de programas de ensino a crianças diagnosticadas com autismo. Acta Comportamentalia, 23, 405-421.

Borba, M. C., Monteiro, P. C. M., Barboza, A. A., Trindade, E. N., \& Barros, R. S. (2015). Efeito de intervenção via cuidadores sobre aquisição de tato com autoclítico em crianças com TEA. Revista Brasileira de Análise do Comportamento, 11, 15-23. doi: 10.18542/rebac.v11i1.3768

Brodhead, M. T., Higbee, T. S., Gerencser, K. R., \& Akers, J. S. (2016). The use of a discriminationtraining procedure to teach mand variability to children with autism. Journal of Applied Behavior Analysis, 49, 34-48. doi: 10.1002/jaba.280

Coelho, C. A. (1990). Acquisition and generalization of simple manual sign grammars by aphasic subjects. Journal of Communication Disorders, 23(6), 383-400. doi: 10.1016/0021-9924(90)90026-U

Correa Gómez, L. E. (2015). Acquisition and generalization of tacts across stimulus modes in children diagnosed with autism spectrum disorder. Master Theses. University of South Florida.

Cuvo, A. J., Klevans, L., Borakove, S., Borakove, L. S., Landuyt, J. V., \& Lutzker, J. R. (1980). A comparison of three strategies for teaching object names. Journal of Applied Behavior Analysis, 13, 249-257. doi: 10.1901/jaba.1980.13-249

Dixon, M. R., Peach, J., Daar, J. H., \& Penrod, C. (2017). Teaching complex verbal operants to children with autism and establishing generalization using the peak curriculum. Journal of Applied Behavior Analysis, 50(2), 317-331. doi: 10.1002/jaba.373

Dupuis, D. L., Lerman, D. C., Tsami, L., \& Shireman, M. L. (2015). Reduction of aggression evoked by sounds using noncontingent reinforcement and time-out. Journal of Applied Behavior Analysis, 48, 669-674. doi: 10.1002/jaba.220

Farber, R. S., Dube, W. V., \& Dickson, C. A. (2016). A sorting-to-matching method to teach compound matching to sample. Journal of Applied Behavior Analysis, 49, 294-307. doi: 10.1002/jaba.290
Ferreira, L. A., Silva, A. J. M., \& Barros, R. S. (2016). Ensino de aplicação de tentativas discretas a cuidadores de crianças com autismo. Perspectivas em Análise do Comportamento, 7, 101-113. doi: 10.18761/pac.2015.034

Frampton, S. E., Wymer, S. C., \& Hansen, B. (2016). The use of matrix training to promote generative language with children with autism. Journal of Applied Behavior Analysis, 49, 869-883. doi: 10.1002/jaba.340

Fiorile, C. A., \& Greer, R. D. (2007). The induction of naming in children with no prior tact responses as a function of multiple exemplar histories of instruction. The Analysis of Verbal Behavior, 23(1), 71.

Gruber, D. J., \& Poulson, C. L. (2016). Graduated guidance delivered by parents to teach yoga to children with developmental delays. Journal of Applied Behavior Analysis, 49, 193-198. doi: 10.1002/jaba.260

Hine, J. F., Ardoin, S. P., \& Foster, T. E. (2015). Decreasing transition times in elementary school classrooms: Using computer-assisted instruction to automate intervention components. Journal of Applied Behavior Analysis, 48, 495-510. doi: 10.1002/jaba.233

Jeffries, T., Crosland, K., \& Miltenberger, R. (2016). Evaluating a tablet application and differential reinforcement to increase eye contact in children with autism. Journal of Applied Behavior Analysis, 49, 182187. doi: $10.1002 /$ jaba.262

Keller, F. S., \& Schoenfeld, W. N. (1950). Principles of Psychology: a systematic text in the science of behavior. New York: Appleton-Century-Crofts.

LeBlanc, L. A., Coates, A. M., Daneshvar, S., Charlop-Christy, M. H., Morris, C., \& Lancaster, B. M. (2003). Using video modeling and reinforcement to teach perspective-taking skills to children with autism. Journal of Applied Behavior Analysis, 36(2), 253-257. doi: 10.1901/jaba.2003.36-253

MacDonald, J., \& Ahearn, W. H. (2015). Teaching observational learning to children with autism. Journal of Applied Behavior Analysis, 48, 800-816. doi: 10.1002/jaba.257

Marzullo-Kerth, D., Reeve, S. A., Reeve, K. F., \& Townsend, D. B. (2011). Using multiple-exemplar training to teach a generalized repertoire of sharing to children with autism. Journal of Applied Behavior Analysis, 44(2), 279-294. doi: 10.1901/jaba.2011.44279

Neef, N. A., Walters, J., \& Egel, A. L. (1984). Establishing generative yes/no responses in developmentally disabled children. Journal of Applied Behavior Analysis, 17(4), 453-460. doi: 10.1901/jaba.1984.17-453

Reeve, S. A., Reeve, K. F., Townsend, D. B., \& Poulson, C. L. (2007). Establishing a generalized repertoire of helping behavior in children with autism. Journal of Applied Behavior Analysis, 40(1), 123-136. doi: 10.1901/jaba.2007.11-05

Shillingsburg, M. A., Kelley, M. E., Roane, H. S., Kisamore, A., \& Brown, M. R. (2009). Evaluation and training of yes-no responding across verbal operants. 
Journal of Applied Behavior Analysis, 42(2), 209-223. doi: 10.1901/jaba.2009.42-209

Silber, J. M., \& Martens, B. K. (2010). Programming for the generalization of oral reading fluency: Repeated readings of entire text versus multiple exemplars. Journal of Behavioral Education, 19(1), 30-46. doi: 10.1007/s10864-010-9099-0

Silva, A. J. M. (2015). Aplicação de tentativas discretas por cuidadores para o ensino de habilidades verbais a crianças diagnosticadas com autismo. Dissertação de mestrado. Programa de Pós-Graduação em Teoria e Pesquisa do Comportamento, Universidade Federal do Pará.

Skinner, B. F. (1953). Science and human behavior. New York: The MacMillan Company.

Stokes, T. F., \& Baer, D. M. (1977). An implicit technology of generalization. Journal of Applied Behavior Analysis, 10, 349-367. doi: 10.1901/jaba.1977.10-349
Sundberg, M. L. (2014) Verbal behavior milestones assessment and placement program: The VB-MAPP. Concord, CA: AVB Press.

Wacker, D. P., Berg, W. K., Berrie, P., \& Iwatta, P. (1985). Generalization and maintenance of complex skills by severely handicapped adolescents following picture prompt training. Journal of Applied Behavior Analysis, 18(4), 329-336. doi: 10.1901/jaba.1985.18329

Wong, C., Odom, S. L., Hume, K. Cox, A. W., Fettig, A., Kucharczyk, S., \& Schultz, T. R. (2014). Evidencebased practices for children, youth, and young adults with Autism Spectrum Disorder. Chapel Hill: The University of North Carolina, Frank Porter Graham Child Development Institute, Autism Evidence-Based Practice Review Group.

Submetido em 04/03/2017 Aceito em 09/08/2017 